\title{
A Systematic Review and Meta-analysis on the Prevalence of Low Birth Weight Infants in Iran
}

\author{
Maryam Sabbaghchi, ${ }^{1}$ Rostam Jalali, ${ }^{2}$ and Masoud Mohammadi $\mathbb{D}^{2}$ \\ ${ }^{1}$ Department of Demography, Faculty of Social Sciences, Yazd University, Yazd, Iran \\ ${ }^{2}$ Department of Nursing, School of Nursing and Midwifery, Kermanshah University of Medical Sciences, Kermanshah, Iran \\ Correspondence should be addressed to Masoud Mohammadi; masoud.mohammadi1989@yahoo.com
}

Received 9 May 2020; Revised 16 July 2020; Accepted 5 October 2020; Published 15 October 2020

Academic Editor: Marco Scioscia

Copyright (C) 2020 Maryam Sabbaghchi et al. This is an open access article distributed under the Creative Commons Attribution License, which permits unrestricted use, distribution, and reproduction in any medium, provided the original work is properly cited.

\begin{abstract}
Background. Low birth weight is a significant index for survival, intrauterine growth, and mortality in infants. Thus, this study is aimed at determining the prevalence of low birth weight in Iranian infants through a systematic review and meta-analysis. Methods. This study was performed by meta-analysis from January 2000 to December 2019. The studies relevant to the topic have been obtained through search in databases of Scopus, ScienceDirect, SID, Magiran, Barakat Knowledge Network System, Medline (PubMed), and Google Scholar. Heterogeneity of the studies has been assessed by the $I^{2}$ index, and data analysis was done using Comprehensive Meta-Analysis software. Results. By investigating 14 articles and 93924 infants, the total prevalence of low birth weight in infants in Iran was achieved at 8.5\% (95\% CI: 7.3-9.9\%) according to the meta-analysis; the most prevalence of low birth weight was in infants in Hamedan at 19.1\% (95\% CI: 21.2-17.2\%) in 2007, and the lowest prevalence of low birth weight was in infants in Tonekabon at 4.2\% (95\% CI: 3.4-5.2\%) in 2005, and also, by increasing the sample size, the prevalence of low birth weight increases, by which the difference is statistically significant $(P<0.05)$. Conclusion. Due to the high prevalence of low birth weight in infants in Iran, health policy-makers must take effective attempts in order to reduce it in infants.
\end{abstract}

\section{Background}

Intrauterine growth restriction (IUGR) refers to a condition in which an unborn baby is smaller than it should be because it is not growing generally inside the womb. Delayed growth puts the baby at risk of specific health problems during pregnancy and delivery and after birth. They include low birth weight; difficulty handling the stresses of vaginal delivery; decreased oxygen levels; hypoglycemia; low resistance to infection; low Apgar scores; meconium aspiration, which can lead to breathing problems; trouble maintaining body temperature; and abnormally high red blood cell count [1-5].

A common cause is a problem with the placenta. The placenta is the tissue that joins the mother and fetus, carrying oxygen and nutrients to the baby and permitting the release of waste products from the baby. The condition can also occur as the result of specific health problems in the mother, such as advanced diabetes; high blood pres- sure or heart disease; infections such as rubella, cytomegalovirus, toxoplasmosis, and syphilis; kidney disease or lung disease; malnutrition or anemia; sickle cell anemia; smoking; drinking alcohol; or abusing drugs [2-6].

Low birth weight is the most important factor determining survival and an essential marker of intrauterine growth. It is also one of the most straightforward and most general health indices in each society and is a crucial index in infant mortality [1-3]. Approximately $16 \%$ of births lead to low birth weight infants annually, by which $70 \%$ of them result in death [4-6]. The World Health Organization named infants with a birth weight lower than 2500 grams as low birth weight (LBW) [7]; mortality in infants with weight lesser than $2500 \mathrm{~g}$ is higher 40 times, and mortality in infants with weight more minor than $1500 \mathrm{~g}$ is higher 200 times more than infants with weight greater than $2500 \mathrm{~g}$ [7].

In addition to spiritual and mental problems, the high costs of caring and treatments of LBW infants at birth are 
borne by their families, who often belonged to vulnerable society segments [8]; out of 25 million infants with LBW born annually, more than $90 \%$ of them are born in developing countries [9]; in the classification of causes for infants' mortality, low birth weight is the second important cause for infant death in $40-80 \%$ cases [6-9].

LBW infants are more susceptible to risks such as cerebral palsy, mental retardation, neural defects, pulmonary diseases, sudden death syndrome, and complications caused by hospitalization at intensive care units in comparison to healthy weight infants [10-12]. In the conducted studies in various regions in Iran, the prevalence of LBW was reported as $11.8 \%$ in Qom [13], 19.1\% in Hamedan [14], 8.6\% in Tehran [15], 7.7\% in Babol [16], and 6.9\% in Guilan [17], which is indicating inconsistency and ambiguities in the prevalence of LBW in infants in Iran.

Since low birth weight increases the rate of mortality, disability, and many diseases in childhood and adulthood, and since performing interventional studies to reduce the prevalence of low birth weight in infants require accurate and consistent information to prevent problems and complications of LBW, the question of this research is on the rate of the total prevalence of LBW in infants in Iran. This study is aimed at determining the incidence of LBW in infants in Iran through a systematic review and metaanalysis.

\section{Methods}

2.1. Search Strategy. This was a systematic review and metaanalysis done by extraction of results of conducted studies in the context of the prevalence of LBW in infants in Iran and consisted of articles published in domestic and foreign journals and literature review in databases of ScienceDirect, Scopus, SID, Magiran, Medline (PubMed), Barakat Knowledge Network System, and Google Scholar from Jan 2000 to Dec 2019. The literature review has been performed by using keywords of low-birth weight, infants, and Iran and also probable combinations in English and Persian language. Therefore, literature review in Persian-language databases has been done by using mentioned Persian-language keywords, and for literature review in English-language databases, the English equivalent words of low birth weight, birth, neonates, and Iran were used, and also, for searching in Google Scholar, every two words in Persian and English language have been used, and logical operators of AND and OR in combination have been applied in order to have complete access to all the articles. Therefore, the operator of OR has been applied to investigate common names in the cases such as (Low birth weight OR Low-Birth-Weight Infant OR Infant LBW) and (neonates OR Infant OR Newborn), and also, the operator of AND has been applied between keywords of (Low birth weight AND neonates) through matching the words in the MeSH browser.

2.2. Inclusion Criteria and Evaluation of Articles. Firstly, all the articles were gathered by using selected keywords, and after finishing the literature review, a list consisting of articles' abstracts was prepared.
After hiding the characteristics of articles, including the journal name and authors, the full texts have been provided to reviewers. Each article was studied by two independent reviewers. In case of rejection, the reason for refusal has been mentioned. In case of a lack of consensus between the two reviewers, the article was reviewed by a third reviewer, and the third reviewer's opinion was considered.

Articles in English and Persian language with crosssectional design in the context of prevalence and frequency of LBW in Iranian infants were eligible to be enrolled in the study, and other review studies, case-control studies, cohort, and interventional studies were excluded from the article list. In this study, a review of gray literature, evidence, and documents not printed and published due to any reason was aimed at searching keywords in Google search and reviewing topic-relevant websites.

Then, investigated studies were evaluated based on the 4stage process of PRISMA 2009 consisting of identification of articles, screening, assessing the criteria for accepting the articles, and finally assessing the included articles in the meta-analysis.

2.3. Qualitative Assessment. In order to assess the studies, the STROBE checklist was used. This checklist consisted of 22 sections, by which 18 items of it are general and are used for all observational studies including cohort, case-control, and cross-sectional studies, and 4 items of it are specified, which is related to the type of study, and include various aspects of methodology such as the study's objectives, appropriate determination of sample size, type of study, sampling method, study population, data gathering method, description of variables, the way samples were evaluated, data gathering tools, objectives assessed in the study, applied statistical test, and report of the results. Accordingly, the maximum score for qualitative assessment was considered 32, and articles with scores of lesser than 14 were considered poor based on assessment and evaluation and were excluded from the study.

2.4. Statistical Analysis. The prevalence of LBW in infants for each study conducted in Iran was achieved; heterogeneity of the studies has been investigated by the $I^{2}$ test. Generally, heterogeneity has classified into three classes: heterogeneity less than 25\% (low heterogeneity), between 25 and 75\% (moderate heterogeneity), and more than $75 \%$ (high heterogeneity). Data were analyzed by Comprehensive Meta-Analysis software (Biostat, Englewood, NJ, USA, version 3 ). The probability of publication bias in the results has been evaluated by a Funnel plot and by using the Egger test at the significance level of 0.05, and also, to assess the impacts of active potential factors in the heterogeneity of studies, a metaregression test has been used in two elements of sample size and the year the study was performed in.

\section{Results}

The results are based on conducted researches on the prevalence of LBW in Iranian infants, consisting of published 


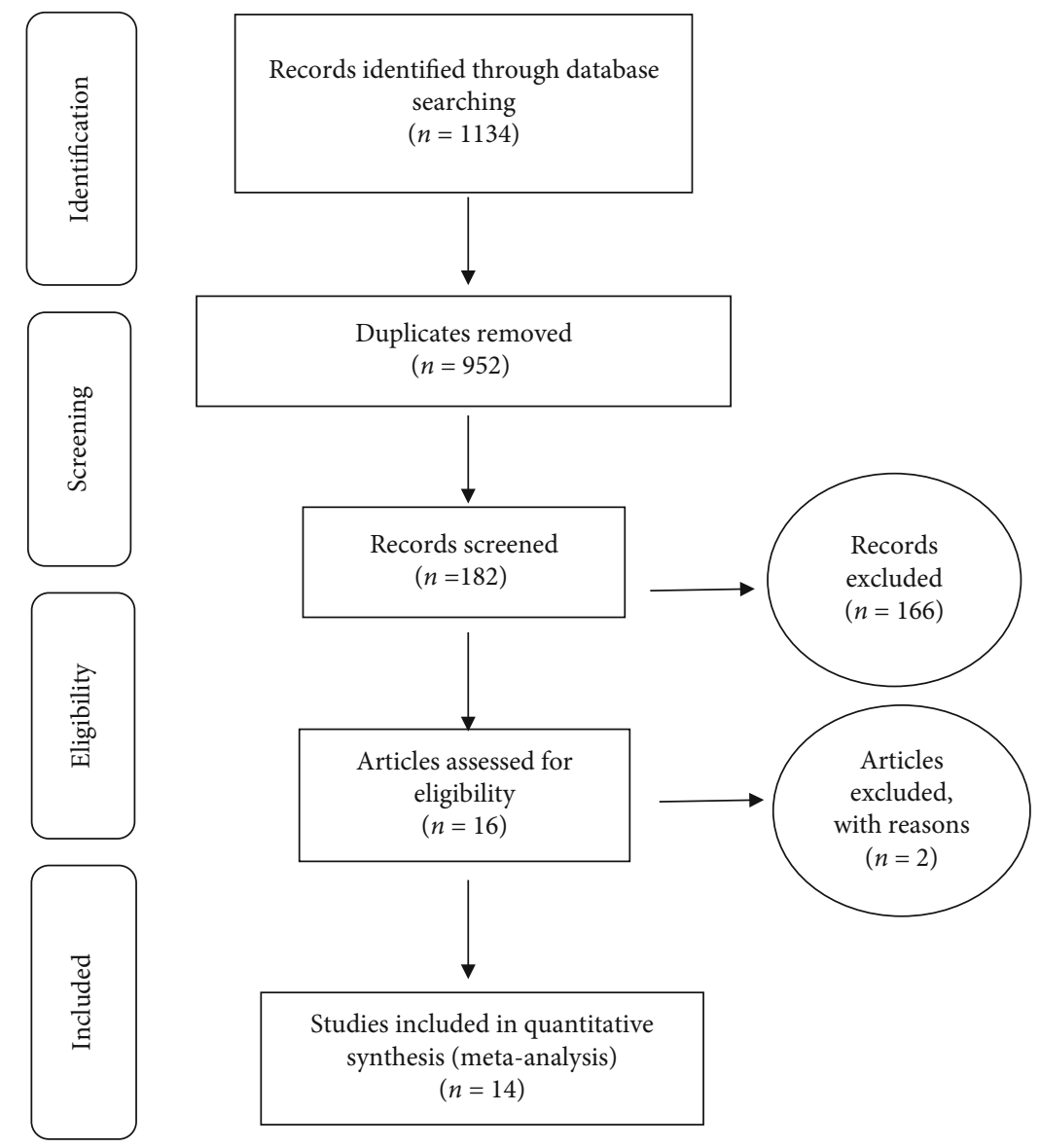

FIGURE 1: The flowchart on the stages of including the studies in the systematic review and meta-analysis (PRISMA 2009).

articles in domestic and foreign journals. Literature review was done to 95 articles in databases of Magiran, SID, and Barakat Knowledge Network System, 185 articles in Medline (PubMed), 321 articles in ScienceDirect, 72 articles in Scopus, and 461 articles in Google Scholar, and 1134 articles were obtained based on the 4-stage process of PRISMA 2009 (Figure 1). Then, 182 eligible articles based on primary investigations after omitting 952 duplicate articles remained. Finally, 166 irrelevant articles have been omitted, and after secondary evaluation, two articles were also omitted due to lack of access to the abstract and full text and low quality of articles; 14 articles were finally entered into the metaanalysis process (Table 1) [13-26].

3.1. Evaluation of Heterogeneity and Publication Bias. Heterogeneity in articles was assessed by the $I^{2}$ test, and based on this test, the value of $96 \%$ was achieved and is a representative of high heterogeneity in entered studies; therefore, the random effects model was used to combine the studies' results; obtained results from evaluation of publication bias in the studies were assessed by the Egger test (Figure 2) by which the bias was not statistically significant $(P=0.537)$.

The total number of samples entered in the study was 93924 infants, and the overall prevalence of LBW in Iranian infants based on meta-analysis has been achieved at $8.5 \%$ (95\% CI: 7.3-9.9\%). The highest incidence of LBW was in infants in Hamadan at 19.1\% (95\% CI: 17.2-21.2\%) [14] in 2007, and the lowest prevalence of LBW was in infants in Tonekabon at $4.2 \%$ (95\% CI: $3.4-5.2 \%)$ [21] in 2005 (Figure 3). In this figure, the prevalence of LBW is shown based on the random effects model, by which the black square is the rate of prevalence, and the length of the segment by which the square lays on is the confidence interval for each study; the diamond sign shows the prevalence in the whole country for all the studies.

3.2. Metaregression Test. To investigate the impacts of active potential factors in the heterogeneity of prevalence of LBW in infants in Iran, a meta-regression test has been used on two factors of sample size and year of investigation (Figures 4 and 5). Based on Figure 4, by increasing the sample size, the total prevalence of LBW in infants in Iran also increases, by which this difference is statistically significant $(P<0.05)$. Figure 5 also reported that by increasing the year of investigation, the total prevalence of LBW in infants in Iran also increases, by which this difference is not statistically significant $(P=0.820)$.

\section{Discussion}

Based on the results obtained from this study and investigations on 93924 infants, the total prevalence of LBW in 
TABle 1: Specifications of studies entered in the study.

\begin{tabular}{|c|c|c|c|c|c|}
\hline Row & Author (references) & Publication year & Area & Sample size & Prevalence \\
\hline 1 & Karimian et al. [13] & 2003 & Qom & 1972 & 11.8 \\
\hline 2 & Eghbalian [14] & 2007 & Hamedan & 1500 & 19.1 \\
\hline 3 & Tootoonchi [15] & 2007 & Tehran & 905 & 8.6 \\
\hline 4 & Zahed Pasha et al. [16] & 2004 & Babol & 2228 & 7.7 \\
\hline 5 & Zarbakhsh et al. [17] & 2012 & Gilan & 325 & 6.9 \\
\hline 6 & Delaram and Ahmadi [18] & 2008 & Shahr-e-kord & 5102 & 8.5 \\
\hline 7 & Bahrami et al. [19] & 2014 & Qazvin & 3076 & 6.7 \\
\hline 8 & Sobhi et al. [20] & 2013 & Mashhad & 7763 & 6.1 \\
\hline 9 & Hosseini et al. [21] & 2005 & Tonekabon & 2016 & 4.2 \\
\hline 10 & Shadzie et al. [22] & 2000 & Isfahan & 848 & 6 \\
\hline 11 & Momeni et al. [23] & 2017 & Kerman & 60273 & 9.4 \\
\hline 12 & Golestan et al. [24] & 2011 & Yazd & 5897 & 8.8 \\
\hline 13 & Roudbari et al. [25] & 2007 & Zahedan & 1109 & 11.8 \\
\hline 14 & Talebian and Afrouz [26] & 2010 & Isfahan & 910 & 9.5 \\
\hline
\end{tabular}

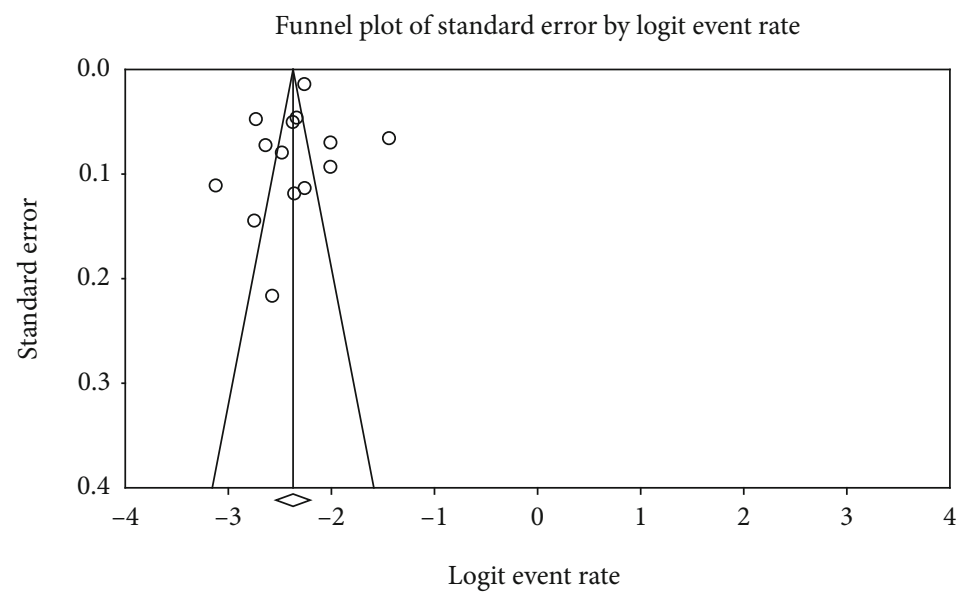

FIgURE 2: Funnel plot of results on the prevalence of low birth weight in Iranian infants.

infants in Iran according to the meta-analysis was achieved at $8.5 \%$. The World Health Organization (WHO) reported incidence of LBW in sub-Saharan Africa at $15 \%$, in the Middle East and northern Africa at $11 \%$, in East Asian countries at $10 \%$, in southern Asia at 33\%, in Latin America at 9\%, in developed countries at $6 \%$, and developing countries and the whole world at $18 \%$ and $17 \%$, respectively [27-30].

Other studies show that the prevalence of LBW inborn infants was $15 \%$ in Kenya, $8 \%$ in Ethiopia, $13.6 \%$ in Saudi Arabia, and 6\% in the United States [31].

The studies showed that most of the LBW infants are with intrauterine growth retardation, which can be due to nutritional deficiency, low maternal age, and lack of pregnancy care $[32,33]$, and other researches show that small maternal age (less than 18 years) can contribute to the birth of LBW infant [34].

These studies report that factors such as trauma to the mother's abdomen leading to preterm labor, membrane rup- ture, placental abruption or detachment, and uterine detachment and also factors such as nicotine and tobacco and alcohol use, low economic status of the family, low maternal weight, anemia, inappropriate diet, sexually transmitted diseases, and mental diseases can be of most important factors leading to LBW in infants [33-35].

The main symptom of IUGR is a small size for gestational age baby. Specifically, the baby's estimated weight is below the 10th percentile or less than that of $90 \%$ of babies of the same gestational age. Depending on the cause of IUGR, the baby may be small all over or look malnourished. They may be weak and pale and have loose, dry skin. The umbilical cord is often thin and dull instead of thick and shiny [33-35].

LBW's importance is due to the birth of girls with LBW, by which they are going to be mothers with a higher probability of giving birth to infants with LBW. In other words, delivery of LBW infants has transmitted intergeneration [36-39]. 


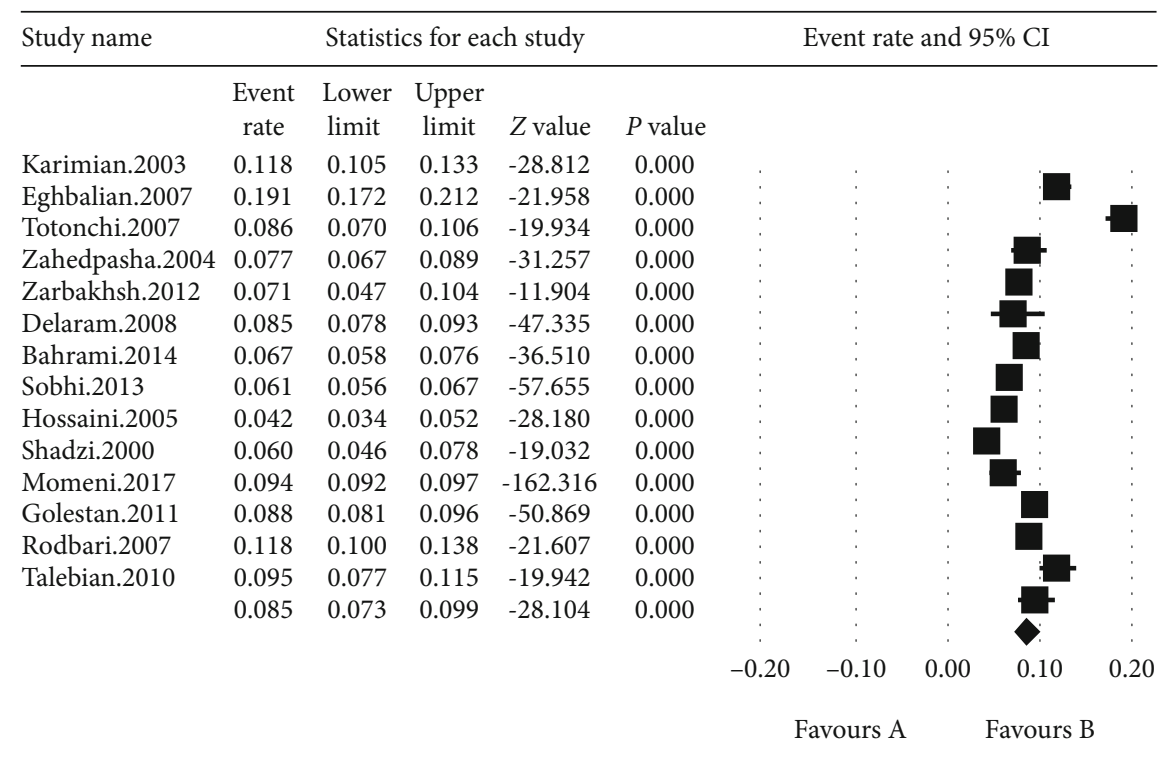

Meta-analysis

FIgURE 3: Total prevalence of LBW in Iranian infants based on the random effects model.

Regression of sample on logit event rate

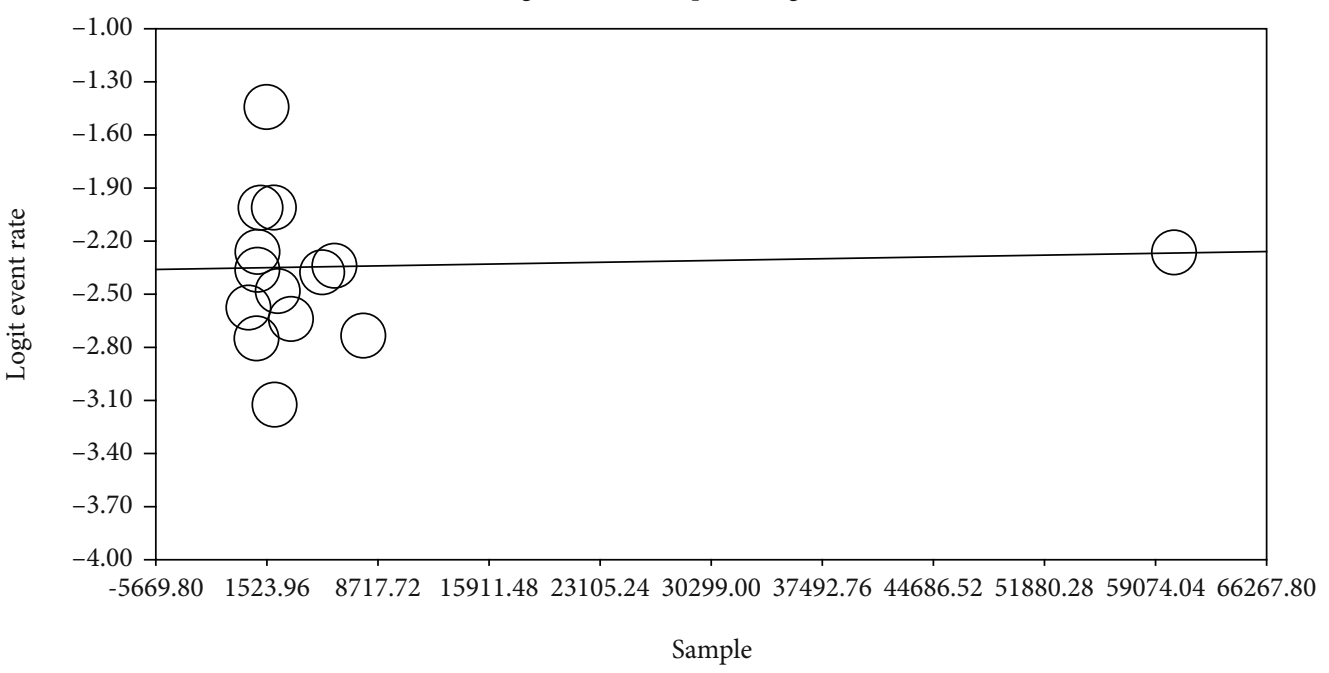

FIGURE 4: Metaregression chart on the prevalence of LBW in infants in Iran divided into sample size.

This condition in various conducted studies in low economic status or areas showed a higher prevalence of LBW so that the incidence of LBW was $39.1 \%$ in India's slum area, $36.8 \%$ in impoverished regions in Bangladesh, and $20.9 \%$ in rural areas of most Asian countries [36-41].

Due to the relatively high prevalence of LBW in Iran, it is necessary to increase the maternal health rate through care processes before and after labor, appropriate nutrition, and prevention of iron deficiency anemia and also through an increase in awareness of parents and mothers by mass media and developing care programs for mothers during pregnancy, and obligation on referral to health centers for mothers and implementing pregnancy care provide the basis for a decline in LBW in infants in-country, and also by equipping and promoting quality of intensive care units in the country, the fundamental basis for hospitalization and treatment of premature and LBW infants would be provided.

4.1. Limitation. The most important limitations of the current study are the lack of access to the full texts of articles and the low quality of some articles.

\section{Conclusions}

Given the high prevalence of LBW in infants in Iran, health policy-makers must produce effective policies to take 


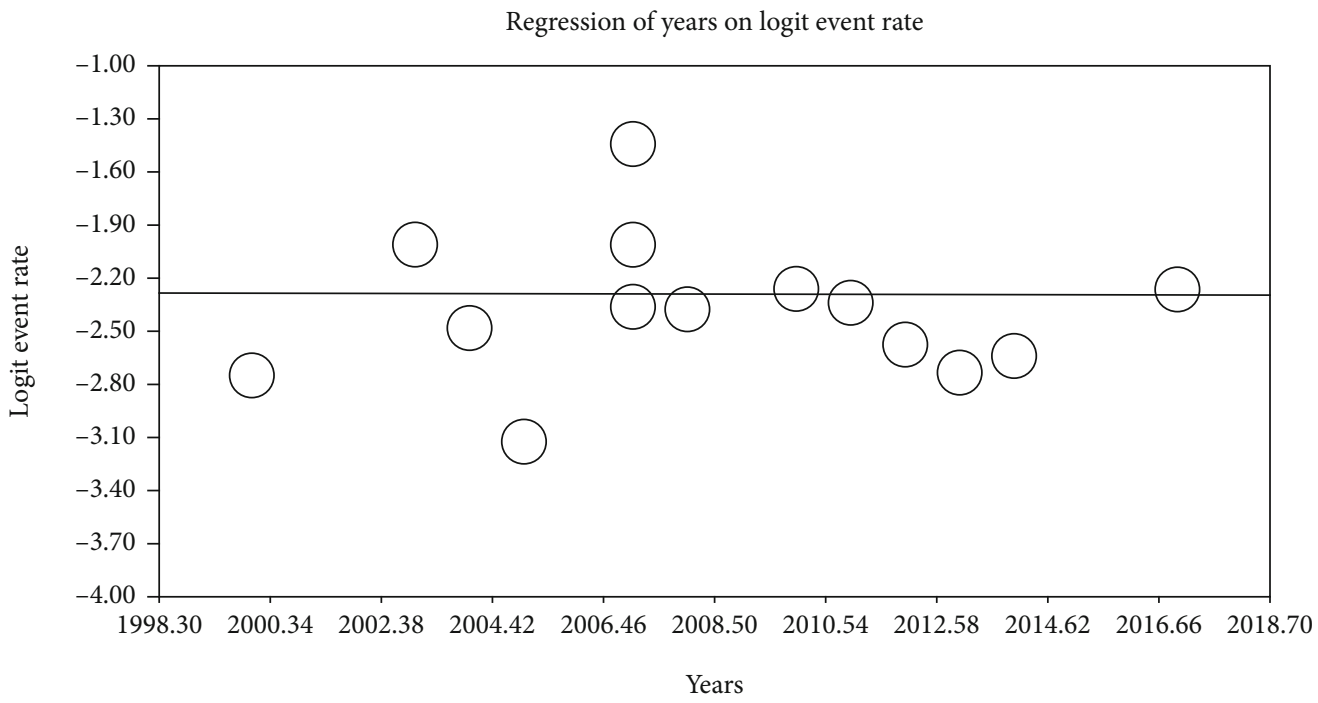

Figure 5: Metaregression chart on the prevalence of LBW in infants in Iran divided into year of investigation.

sufficient management attempts, including periodical care for pregnant mothers and infants.

\section{Abbreviations}

\author{
WHO: $\quad$ World Health Organization \\ LBW: $\quad$ Low birth weight \\ SID: $\quad$ Scientific Information Database \\ STROBE: STrengthening the Reporting of OBservational \\ studies in Epidemiology for cross-sectional study \\ PRISMA: Preferred Reporting Items for Systematic Reviews \\ and Meta-Analyses.
}

\section{Data Availability}

Datasets are available through the corresponding author upon reasonable request.

\section{Ethical Approval}

Ethics approval was received from the ethics committee of the Deputy for Research and Technology, Kermanshah University of Medical Sciences (IR.KUMS.REC.1399.610).

\section{Conflicts of Interest}

The authors declare that they have no conflict of interest.

\section{Authors' Contributions}

MS, MM, and RJ contributed to the design and statistical analysis and prepared the manuscript. All authors have read and approved the content of the manuscript.

\section{Acknowledgments}

The authors thank the faculty members of the Faculty of Nursing and Midwifery, Kermanshah University of Medical Sciences. The study was funded by the Deputy for Research and Technology, Kermanshah University of Medical Sciences (990537).

\section{References}

[1] B. Guyer, S. Ma, H. Grason et al., "Early childhood health promotion and its life course health consequences," Academic pediatrics, vol. 9, no. 3, pp. 142-9.e71, 2009.

[2] G. Alexander, M. Wingate, J. Mor, and S. Boulet, "Birth outcomes of AsianIndian-Americans," International Journal of Gynecology \& Obstetrics, vol. 97, no. 3, pp. 215-220, 2007.

[3] J. Marchi, M. Berg, A. Dencker, E. K. Olander, and C. Begley, "Risks associated with obesity in pregnancy, for the mother and baby: a systematic review of reviews," Obesity Reviews, vol. 16, no. 8, pp. 621-638, 2015.

[4] A. R. Bischoff, A. K. Portella, C. Paquet et al., "Low birth weight is associated with increased fat intake in school-aged boys," British Journal of Nutrition, vol. 119, no. 11, pp. 12951302, 2018.

[5] Z. Z. Shen, Y. W. Wang, S. Ma et al., "Risk factors for preterm birth, low birth weight and small for gestational age: a prospective cohort study," Zhonghua Liu Xing Bing Xue Za Zhi, vol. 40, no. 9, pp. 1125-1129, 2019.

[6] K. Moser, L. Li, and C. Power, "Social inequalities in low birth weight in England and Wales: trends and implications for future population health," Journal of Epidemiology and Community Health, vol. 57, no. 9, pp. 687-691, 2003.

[7] R. Kliegman, Nelson Textbook of Pediatrics, Saunders Elsevier, Philadelphia, 2007.

[8] A. Garshasbi and N. Fallah, "Maternal hematocrite level and risk of low birth weight and preterm delivery (persion)," Tehran University Medical Journal TUMS Publications, vol. 64, no. 4, pp. 87-93, 2006.

[9] M. Mirza Rahimi, H. Saadati, M. Brak, N. Abasgholizadeh, and A. Azami, "The incidence and causes of low birth weight in Ardebil hospitals," Iranian Journal of Ardabil University of Medical Sciences, vol. 9, no. 1, pp. 69-79, 2008.

[10] B. J. Stoll and R. M. Kliegtman, "Overview of mortality and morbidity," in Nelson Textbook of Pediatrics, R. E. Behrman, 
R. M. Kliegtman, and H. B. Jenson, Eds., pp. 519-523, Saunders company, Philadelphia, 17th Ed. edition, 2004.

[11] E. Mansour, A. N. Eissa, L. M. Nofal, I. Kharboush, and A. A. Reda, "Morbidity and mortality of low-birth-weight infants in Egypt," Eastern Mediterranean Health Journal, vol. 11, no. 4, pp. 723-731, 2005.

[12] M. C. Hoffman, S. Jeffers, J. Carter, L. Duthely, A. Cotter, and V. H. Gonzalez-Quintero, "Pregnancy at or beyond age 40 years is associated with an increased risk of fetal death and other adverse outcomes," American Journal of Obstetrics and Gynecology, vol. 196, no. 5, pp. e11-e13, 2007.

[13] S. Karimian, M. Molamohammadi, and G. Jandaghi, "Prevalence and factors associated with low-weight newborns in the maternity Qom Province in 2000," Iranian Journal of Faiz, vol. 27, no. 3, pp. 76-80, 2004.

[14] F. Eghbalian, "Low birth weight causes survey in neonates Iranian," Journal of Pediatrics, vol. 17, no. 1, pp. 27-33, 2007.

[15] P. Tootoonchi, "Low birth weightamong newborn infants at Tehran Hospitals," Iranian Journal of Pediatrics, vol. 17, no. 2, pp. 186-192, 2007.

[16] Y. Zahed Pasha, M. R. Esmaeili Dooki, M. Haji Ahmadi et al., "Effect of risk factors on low birth weight neonates," Journal of Babol University of Medical Sciences, vol. 6, no. 2, pp. 18-24, 2004.

[17] M. R. Zarbakhsh Bhari, S. Hoseinian, A. AfroozGh, and H. A. Hooman, "Prevalence of low birth weight and comparison of many biological characteristics of low birth weight -newborns, mothers with those of normal weight-newborns' counterparts," Journal of Guilan University of Medical Sciences, vol. 21, no. 81, pp. 37-44, 2011.

[18] M. Delaram and A. R. Ahmadi, "Prevalence of low birth weight and its related factors in Shahr-e-Kord," Reproduction and Infertility Journal, vol. 87, no. 3, pp. 263-270, 2008.

[19] N. Bahrami, M. A. Soleimani, Y. H. Chan, R. Masoudi, and L. Rabiei, "Study of some determinants of birth weight in Qazvin," Journal of Clinical Nursing and Midwifery, vol. 3, no. 4, pp. 56-64, 2014.

[20] A. S. Sobhi, M. Kazemi, and D. A. Rezaie, "The prevalence of low birth weight in newborns and its correlation with major causes of neonatal mortality, during 2008-2011 in Fariman city, Iran," The Journal of Research Committee of Students at Sabzavar University of Medical Sciences, vol. 18, no. 3,4, pp. 7-13, 2013.

[21] S. Hosseini, M. Bahadori, and H. Fallah Bagher Shaidaei, "Incidence of low birth weight and associated risk factors during March 2002-2003 in Tonekabon, Iran," Journal of Mazandaran University of Medical Sciences, vol. 15, no. 49, pp. 110-113, 2005.

[22] S. Shadzie, Z. Mohammadzadeh, F. Mostafavie, and A. Hassanzadeh, "Privalence of low birth weight and their relation with some of maternal risk factors in Isfahan," Journal of Guilan University of Medical Sciences, vol. 9, no. 33 and 34, pp. 55-61, 2000.

[23] M. Momeni, M. Danaei, A. J. N. Kermani et al., "Prevalence and risk factors of low birth weight in the Southeast of Iran," International Journal of Preventive Medicine, vol. 8, no. 1, p. 12, 2017.

[24] M. Golestan, S. Akhavan Karbasi, and R. Fallah, "Prevalence and risk factors for low birth weight in Yazd, Iran," Singapore Medical Journal, vol. 52, no. 10, pp. 730-733, 2011.

[25] M. Roudbari, M. Yaghmaei, and M. Soheili, "Prevalence and risk factors of low-birth-weight infants in Zahedan, Islamic
Republic of Iran," Eastern Mediterranean Health Journal, vol. 13, no. 4, pp. 838-845, 2007.

[26] M. H. Talebian and G. A. Afrouz, "The relationship between biological, psychological-cognitive and social-cultural characteristics of parents with infant's birth weight in Isfahan Province," Health System Research, vol. 6, no. 2, pp. 194-204, 2011.

[27] UNICEF, The state of the world's children 1999, United Nation Children's Fund, WHO, 1999.

[28] M. S. Kramer, "Determinants of low birth weight: methodological assessment and meta-analysis," Bulletin of the World Health Organization, vol. 65, no. 5, pp. 663-737, 1987.

[29] S. Brown, J. Yelland, G. Sutherland, P. Baghurst, and J. Robinson, "Stressful life events, social health issues and low birthweight in an Australian population-based birth cohort: challenges and opportunities in antenatal care," BMC Public Health, vol. 11, no. 1, p. 196, 2011.

[30] A. Zohoor, "Associated with overweight in the first days of pregnancy the baby at birth," Iranian Journal of Fertility and Infertility, vol. 2, no. 2, pp. 33-39, 2003.

[31] C. C. Murphy, B. Schei, T. L. Myhr, and J. du Mont, "Abuse: a risk factor for low birth weight? A systematic review and metaanalysis," Canadian Medical Association Journal, vol. 164, no. 11, pp. 1567-1572, 2001.

[32] O. Aminian, A. Rashedi, and K. Sadegh Niat Hagigi, "Shift effect on neonatal birth weight and gestational age in women," Occupation Medicine Journal, vol. 2, no. 1, pp. 8-13, 2010.

[33] M. H. Fallah, G. A. Afrouz, and G. A. Haidari, "Factors affecting birth weight infants in Yazd .1386," Iranian Journal of Toloe Behdasht, vol. 3, no. 4, pp. 57-63, 2008.

[34] A. Hajivandi and H. Ghaedi, "Waterpipe smoking during pregnancy and its effect on low birth weight and infant health characteristics," Iranian Journal of Semnan University of Medical Sciences, vol. 7, no. 1,2, pp. 1-7, 2006.

[35] H. Hoursan, A. Mehdizadeh, and R. Hoursan, "Relationship between fundal height, abdominal circumference, body mass index and infant weight," Iranian Journal of Iranian University of Medical Sciences, vol. 12, no. 46, pp. 401-408, 2006.

[36] M. D. Gisselmann, "Education, infant mortality, and low birth weight in Sweden 1973-1990: emergence of the low birth weight paradox," Scandinavian Journal of Public Health, vol. 33, no. 1, pp. 65-71, 2016.

[37] P. Chhabra, A. K. Sharma, V. L. Grover, and O. P. Aggarwal, "Prevalence of low birth weight and its determinants in an urban resettlement area of Delhi," Asia-Pacific Journal of Public Health, vol. 16, no. 2, pp. 95-98, 2016.

[38] J. Valero de Bernabé, T. Soriano, R. Albaladejo et al., "Risk factors for low birth weight: a review," European Journal of Obstetrics, Gynecology, and Reproductive Biology, vol. 116, no. 1, pp. 3-15, 2004.

[39] S. Wannous and S. Arous, "Incidence and determinants of low birth weight in Syrian government hospitals," Eastern Mediterranean Health Journal, vol. 7, no. 6, pp. 966-974, 2001.

[40] C. S. Wang and P. Chou, "Risk factors for low birth weight, among first-time mothers in southern Taiwan," Journal of the Formosan Medical Association, vol. 100, no. 3, pp. 168172, 2001.

[41] N. Nahar, S. Afroza, and M. Hossain, "Incidence of low birth weight in three selected communities of Bangladesh," Bangladesh Medical Research Council Bulletin, vol. 24, no. 2, pp. 4954, 1998. 